\title{
A note on homomorphisms between products of algebras
}

\author{
Ivan Chajda, Martin Goldstern and Helmut Länger
}

\begin{abstract}
Let $\mathcal{K}$ be a congruence distributive variety and call an algebra hereditarily directly irreducible (HDI) if every of its subalgebras is directly irreducible. It is shown that every homomorphism from a finite direct product of arbitrary algebras from $\mathcal{K}$ to an HDI algebra from $\mathcal{K}$ is essentially unary. Hence, every homomorphism from a finite direct product of algebras $\mathbf{A}_{i}(i \in I)$ from $\mathcal{K}$ to an arbitrary direct product of HDI algebras $\mathbf{C}_{j}(j \in J)$ from $\mathcal{K}$ can be expressed as a product of homomorphisms from $\mathbf{A}_{\sigma(j)}$ to $\mathbf{C}_{j}$ for a certain mapping $\sigma$ from $J$ to $I$. A homomorphism from an infinite direct product of elements of $\mathcal{K}$ to an HDI algebra will in general not be essentially unary, but will always factor through a suitable ultraproduct.
\end{abstract}

Mathematics Subject Classification. 06B05.

Keywords. Direct product of chains, Homomorphism, Essentially unary mapping, Ultrafilter.

\section{Introduction}

Let $\mathbf{A}_{i}, i \in I$, and $\mathbf{B}_{j}, j \in J$, be algebras of the same type and $f$ a homomorphism from $\prod_{i \in I} \mathbf{A}_{i}$ to $\prod_{j \in J} \mathbf{B}_{j}$. For every $k \in J$ let $p_{k}$ denote the projection from $\prod_{j \in J} \mathbf{B}_{j}$ onto $\mathbf{B}_{k}$ and $f_{k}:=p_{k} \circ f$. More generally, for any $J_{0} \subseteq J$ we let $p_{J_{0}}: \prod_{j \in J} \mathbf{B}_{j} \rightarrow \prod_{j \in J_{0}} \mathbf{B}_{j}$ be the canonical projection map. It is evident that $f=\left(f_{j}: j \in J\right)$. Hence, the task of describing $f$ is reduced to the task of describing the homomorphisms $f_{k}$ from $\prod_{i \in I} \mathbf{A}_{i}$ to $\mathbf{B}_{k}$.

Presented by G. Czédli.

I. Chajda and H. Länger gratefully acknowledge support of this research by ÖAD, project CZ 04/2017, as well as by IGA, project PřF 2018 012. M. Goldstern gratefully acknowledges support by the Austrian Science Fund (FWF), project I 3081-N35. H. Länger gratefully acknowledges support of this research by the Austrian Science Fund (FWF), project I 1923N25. 
In [3] the authors solve this problem for the case that the algebras $\mathbf{A}_{i}$ and $\mathbf{B}_{j}$ are conservative median algebras and the index sets are finite. More generally, Couceiro et al. [2] considers the case that the $\mathbf{A}_{i}$ are median algebras and the $\mathbf{B}_{j}$ are tree-median algebras. It turns out that the method developed in $[2,3]$ can be further generalized to lattices. Let us note that every distributive lattice is a median algebra (but not conversely). We are even able to extend this result to arbitrary lattices $\mathbf{A}_{i}$ provided the $\mathbf{B}_{j}$ are chains. For lattice concepts used in the rest of the paper the reader is referred to the monographs $[1,5]$.

We call a mapping $f: \prod_{i \in I} A_{i} \rightarrow C$ essentially unary if there exists an $i_{0} \in I$ and a mapping $g: A_{i_{0}} \rightarrow C$ with $g \circ p_{i_{0}}=f$. In this case we say that " $f$ depends only on the $i_{0}$-th coordinate", or that " $f$ factors through $p_{i_{0}}$ ".

From $f=g \circ p_{i_{0}}$ it easily follows that $g$ is a homomorphism if and only if $f$ is.

\section{Fraser-Horn property and HDI algebras}

Definition 2.1. A class $\mathcal{K}$ of algebras has the Fraser-Horn property if there are no skew congruences on any product $\mathbf{A}_{1} \times \mathbf{A}_{2}$ with $\mathbf{A}_{1}, \mathbf{A}_{2} \in \mathcal{K}$, or more explicity:

For all $\mathbf{A}_{1}, \mathbf{A}_{2} \in \mathcal{K}$, for every congruence $\theta \in \operatorname{Con}\left(\mathbf{A}_{1} \times \mathbf{A}_{2}\right)$ there are congruences $\theta_{1} \in \operatorname{Con}\left(\mathbf{A}_{1}\right), \theta_{2} \in \operatorname{Con}\left(\mathbf{A}_{2}\right)$ such that $\theta=\theta_{1} \times \theta_{2}$, i.e. $\theta=\left\{\left(\left(x_{1}, x_{2}\right),\left(y_{1}, y_{2}\right)\right) \mid x_{1} \theta_{1} y_{1}, x_{2} \theta_{2} y_{2}\right\}$.

The following lemma is known from [4].

Lemma 2.2. Let $\mathcal{K}$ be a congruence distributive $(C D)$ variety. Then $\mathcal{K}$ has the Fraser-Horn property.

For the rest of the paper we fix a variety $\mathcal{K}$ with the Fraser-Horn property.

We call an algebra non-trivial if its universe contains at least two elements.

Definition 2.3. We call an algebra A hereditarily directly irreducible (HDI) if every subalgebra $\mathbf{B} \leq \mathbf{A}$ is directly irreducible, i.e., is not isomorphic to a direct product of two non-trivial factors.

Fact 2.4. (1) The variety of lattices is congruence distributive.

(2) A lattice is HDI if and only if it is a chain.

Theorem 2.5. Let $\mathcal{K}$ be a variety with the Fraser-Horn property. If $n$ is a positive integer, $\mathbf{A}_{1}, \ldots, \mathbf{A}_{n}$ are in $\mathcal{K}$ and $\mathbf{C} \in \mathcal{K}$ is $H D I$, then every homomorphism $f$ from $\mathbf{A}_{1} \times \cdots \times \mathbf{A}_{n}$ to $\mathbf{C}$ is essentially unary, i.e., factors through one of the projections $p_{i}$.

Proof. Let $\theta=\operatorname{ker}(f)$. By a straightforward generalization of the Fraser-Horn property we know that $\theta=\theta_{1} \times \cdots \times \theta_{n}$, where each $\theta_{i}$ is a congruence on $\mathbf{A}_{i}$.

The homomorphism theorem tells us that $\mathbf{B}^{\prime}:=f\left(\mathbf{A}_{1} \times \cdots \times \mathbf{A}_{n}\right)$ is isomorphic to the direct product $\left(\mathbf{A}_{1} / \theta_{1}\right) \times \cdots \times\left(\mathbf{A}_{n} / \theta_{n}\right)$. By our assumption, $\mathbf{B}^{\prime}$ is directly irreducible, so at most one of these factors can be non-trivial, 
so there is at most one $i$ such that $\mathbf{A}_{i} / \theta_{i}$ has more than one element. So $f$ depends only on the $i$-th coordinate.

Remark 2.6. If $f: \mathbf{A}_{1} \times \mathbf{A}_{2} \rightarrow \mathbf{C}$ is not constant, then there is at most one $i \in\{1,2\}$ such that $f$ factors through $p_{i}$.

As a consequence of the above theorem we obtain the following statement.

Theorem 2.7. If $n$ is a positive integer, $\mathbf{A}_{1}, \ldots, \mathbf{A}_{n} \in \mathcal{K}$, where $\mathcal{K}$ has the Fraser-Horn property, $\left(\mathbf{C}_{j} ; j \in J\right)$ is a non-empty family of HDI algebras in $\mathcal{K}$, and $f$ is a homomorphism from $\mathbf{A}_{1} \times \cdots \times \mathbf{A}_{n}$ to $\prod_{j \in J} \mathbf{C}_{j}$ then there exists a mapping $\sigma: J \rightarrow\{1, \ldots, n\}$ and for every $j \in J$ a homomorphism $g_{j}$ from $\mathbf{A}_{\sigma(j)}$ to $\mathbf{C}_{j}$ such that

$$
f\left(x_{1}, \ldots, x_{n}\right)=\left(g_{j}\left(x_{\sigma(j)}\right) ; j \in J\right)
$$

for all $\left(x_{1}, \ldots, x_{n}\right) \in A_{1} \times \cdots \times A_{n}$.

Proof. Apply Theorem 2.5 to the mappings $f_{j}:=p_{j} \circ f, j \in J$.

Theorem 2.8. Let $\mathcal{K}$ be a variety with the Fraser-Horn property. Let $n, k$ be positive integers and let $\mathbf{A}_{1}, \ldots, \mathbf{A}_{n}, \mathbf{C}_{1}, \ldots, \mathbf{C}_{k}$ be non-trivial $H D I$ algebras in $\mathcal{K}$ and assume $\mathbf{A}_{1} \times \cdots \times \mathbf{A}_{n} \cong \mathbf{C}_{1} \times \cdots \times \mathbf{C}_{k}$. Then $n=k$ and there exists a permutation $\sigma \in \mathrm{S}_{n}$ such that $\mathbf{C}_{i} \cong \mathbf{A}_{\sigma(i)}$ for all $i=1, \ldots, n$.

Proof. Let $f$ denote an isomorphism from $\mathbf{A}_{1} \times \cdots \times \mathbf{A}_{n}$ to $\mathbf{C}_{1} \times \cdots \times \mathbf{C}_{k}$. According to Theorem 2.7, there exist mappings $\sigma$ from $\{1, \ldots, k\}$ to $\{1, \ldots, n\}$ and $\tau$ from $\{1, \ldots, n\}$ to $\{1, \ldots, k\}$, for every $j \in\{1, \ldots, k\}$ a homomorphism $g_{j}$ from $\mathbf{A}_{\sigma(j)}$ to $\mathbf{C}_{j}$ and for every $i \in\{1, \ldots, n\}$ a homomorphism $h_{i}$ from $\mathbf{C}_{\tau(i)}$ to $\mathbf{A}_{i}$ such that

$$
f\left(x_{1}, \ldots, x_{n}\right)=\left(g_{1}\left(x_{\sigma(1)}\right), \ldots, g_{k}\left(x_{\sigma(k)}\right)\right)
$$

for all $\left(x_{1}, \ldots, x_{n}\right) \in A_{1} \times \cdots \times A_{n}$ and

$$
f^{-1}\left(y_{1}, \ldots, y_{k}\right)=\left(h_{1}\left(y_{\tau(1)}\right), \ldots, h_{n}\left(y_{\tau(n)}\right)\right)
$$

for all $\left(y_{1}, \ldots, y_{k}\right) \in C_{1} \times \cdots \times C_{k}$. The injectivity of $f$ implies $k \geq n$ and the injectivity of $f^{-1}$ implies $n \geq k$. This shows $n=k$. Moreover, again since $f$ is injective we have $\sigma \in \mathrm{S}_{\mathrm{n}}$. Finally, the injectivity of $f$ implies the injectivity of $g_{1}, \ldots, g_{n}$ and the surjectivity of $f$ implies the surjectivity of $g_{1}, \ldots, g_{n}$. This shows that $g_{1}, \ldots, g_{n}$ are isomorphisms, i.e. $\mathbf{C}_{i} \cong \mathbf{A}_{\sigma(i)}$ for all $i=1, \ldots, n$.

Corollary 2.9. If an algebra in $\mathcal{K}$ is isomorphic to a finite product of nontrivial HDI algebras, then these factors are uniquely determined up to order and isomorphisms.

Proof. This follows from Theorem 2.8.

We can generalize this to infinite direct products as follows. Recall that an ultrafilter on a set $I$ is a family $U$ of subsets of $I$ which is upwards closed and also closed under intersections such that for all $I_{0} \subseteq I$ exactly one of $I_{0}$, 
$I \backslash I_{0}$ is in $U$. For any family $\left(A_{i}: i \in I\right)$ of sets and any ultrafilter $U$ on $I$ we define the equivalence relation $\sim_{U}$ on $\prod_{i} A_{i}$ by

$$
\left(x_{i}: i \in I\right) \sim_{U}\left(y_{i}: i \in I\right) \Leftrightarrow\left\{i \in I \mid x_{i}=y_{i}\right\} \in U,
$$

and we write $\prod_{i} A_{i} / U$ for the set of equivalence classes, the "ultraproduct of the $A_{i}$ modulo $U$ ". The canonical map from $\prod_{i} A_{i}$ to $\prod A_{i} / U$ is denoted by $\kappa_{U}$. If $\left(\mathbf{A}_{i}\right)_{i \in I}$ is a family of algebras of the same type, then the relation $\sim_{U}$ is a congruence relation on the product $\prod_{i} \mathbf{A}_{i}$.

Theorem 2.10. Let $\mathcal{K}$ be a variety with the Fraser-Horn property. Let $I$ be a non-empty set, and for each $i \in I$ let $\mathbf{A}_{i}$ be an algebra in $\mathcal{K}$. Let $\mathbf{C}$ be an $H D I$ algebra in $\mathcal{K}$, and let $h: \prod_{i \in I} \mathbf{A}_{i} \rightarrow \mathbf{C}$ be a homomorphism which is not constant. Then there is a unique ultrafilter $U$ on I such that $h$ factors through $\kappa_{U}$, i.e., there is a homomorphism $h^{\prime}: \prod_{i \in I} \mathbf{A}_{i} / \sim_{U} \rightarrow \mathbf{C}$ such that $h=h^{\prime} \circ \kappa_{U}$.

In particular: If there is no $i \in I$ such that $h$ factors through $p_{i}$, then $U$ will be a non-principal ultrafilter.

Proof. Let $U$ be defined as the set of all $M \subseteq I$ such that $h$ factors through $p_{M}$, i.e., such that there exists $f_{M}: \prod_{i \in M} \mathbf{A}_{i} \rightarrow \mathbf{C}$ with $h=f_{M} \circ p_{M}$.

It is clear that $U$ is upwards closed, and from Theorem 2.5 and Remark 2.6 we get: If $M_{1} \subseteq I$ and $M_{2}:=I \backslash M_{1}$, then $M_{1} \in U$ and $M_{2} \notin U$ or conversely. As $h$ is not constant, we have $\emptyset \notin U$.

We now show that $U$ is closed under intersections: Given $M_{1}, M_{2} \in U$, then we can write $\prod_{i \in I} \mathbf{A}_{i}$ as the direct product of four factors:

$$
B_{11}=\prod_{i \in M_{1} \cap M_{2}} \mathbf{A}_{i}, B_{10}=\prod_{i \in M_{1} \backslash M_{2}} \mathbf{A}_{i}, B_{01}=\prod_{i \in M_{2} \backslash M_{1}} \mathbf{A}_{i}, B_{00}=\prod_{i \notin M_{1} \cup M_{2}} \mathbf{A}_{i},
$$

with corresponding projections $p_{11}, p_{10}, p_{01}, p_{00}$.

Since none of the sets $M_{1} \backslash M_{2}, M_{2} \backslash M_{1}$, and $I \backslash\left(M_{1} \cup M_{2}\right)$ are in $U, h$ cannot factor through any of $p_{10}, p_{01}$, or $p_{00}$. Hence (by Theorem 2.5 ), $h$ must factor through $p_{11}$, so $M_{1} \cap M_{2} \in U$. So we have shown that $U$ is a filter, and even an ultrafilter.

We now check that $h$ factors through the canonical map $\kappa_{U}: \prod_{i} \mathbf{A}_{i} \rightarrow$ $\prod_{i} \mathbf{A}_{i} / U$. All we have to show is that for all $x \sim_{U} y \in \prod_{i} \mathbf{A}_{i}$ we have $h(x)=$ $h(y)$. Now $x \sim_{U} y$ implies that the set $M:=\{i \mid x(i)=y(i)\}$ is in $U$; by definition of $U$, there is some $f_{M}$ with $h=f_{M} \circ p_{M}$, so we get $h(x)=$ $f\left(p_{M}(x)\right)=f\left(p_{M}(y)\right)=h(y)$.

Finally, we show that $U$ is unique. So let $U^{\prime}$ be an ultrafilter such that $h$ factors through $\kappa_{U^{\prime}}$. It is enough to show $U^{\prime} \subseteq U$ :

Let $M \in U^{\prime}$, and let $U^{\prime} \uparrow M:=\left\{N \cap M \mid N \in U^{\prime}\right\}$ be the restriction of $U^{\prime}$ to $M$. The map $\kappa_{U^{\prime}}$ can be written as $\kappa_{U^{\prime}}=\kappa_{U^{\prime} \uparrow M} \circ p_{M}$; as $h$ factors through $\kappa_{U^{\prime}}, h$ also factors through $p_{M}$, so $M \in U$.

Remark 2.11. Theorem 2.5 was used in the proof of Theorem 2.10; but we can also view Theorem 2.5 as a special case of Theorem 2.10, as any ultrafilter on a finite index set must be principal. 


\section{Lattices}

Theorem 2.10 is in some sense best possible, in the sense that homomorphisms from an infinite product $\prod_{i} \mathbf{A}_{i}$ into an HDI algebra will in general not factor through any single projection $p_{j}$, as the following example shows.

Example 3.1. Let $U$ be an ultrafilter on the infinite index set $I$, and for each $i \in I$ let $\mathbf{A}_{i}$ be the 2-element lattice $\{0,1\}$. Then the ultraproduct $\prod_{i \in I} \mathbf{A}_{i} / U$ is again the 2-element lattice.

Identifying $\prod_{i} \mathbf{A}_{i}$ with the power set lattice $(P(I), \cup, \cap)$, the canonical map $\kappa_{U}: P(I) \rightarrow\{0,1\}$ maps each element of $U$ to 1 and everything else to 0 . If $U$ is a non-principal ultrafilter, then $h_{U}$ does not factor through any projection.

This example can be generalized to any Fraser-Horn variety where the class of HDI algebras is described by a set of first order formulas: If $\prod_{i} \mathbf{A}_{i}$ is a product of algebras, and $\left(h_{i}: i \in I\right)$ is a family of homomorphisms $h_{i}: \mathbf{A}_{i} \rightarrow \mathbf{C}_{i}$, where each $\mathbf{C}_{i}$ is HDI, then the family $\left(h_{i}: i \in I\right)$ naturally defines a homomorphism $h: \prod_{i} \mathbf{A}_{i} \rightarrow \prod_{i} \mathbf{C}_{i}$.

If $U$ is an ultrafilter on $I$, then the algebra $\mathbf{C}:=\prod_{i} \mathbf{C}_{i} / U$ is again HDI (as $\mathbf{C}$ satisfies all first order statements that are true in each $\mathbf{C}_{i}$ ). Let $\kappa_{U}^{C}: \prod_{i} \mathbf{C}_{i} \rightarrow \prod_{i} \mathbf{C}_{i} / U$ and $\kappa_{U}^{A}: \prod_{i} \mathbf{A}_{i} \rightarrow \prod_{i} \mathbf{A}_{i} / U$ be the canonical maps. Then the map $\bar{h}:=\kappa_{U}^{C} \circ h: \prod_{i} \mathbf{A}_{i} \rightarrow \mathbf{C}$ trivially factors through $\kappa_{U}^{A}$, i.e., there is $h^{\prime}: \prod_{i} \mathbf{A}_{i} / U \rightarrow \mathbf{C}$ with $\bar{h}=h^{\prime} \circ \kappa_{U}^{A}$. By the uniqueness claim in Theorem 2.10, we see that $U$ is the set of all $M \subseteq I$ such that $\bar{h}$ factors through $p_{M}$. So if $U$ is non-principal, then $\bar{h}$ does not factor through any $p_{i}$.

Fact 3.2. Let $\mathbf{A}$ be a lattice. Then the following are equivalent:

- There is a non-constant homomorphism from $\mathbf{A}$ into a chain.

- There is a non-constant homomorphism from $\mathbf{A}$ into the 2-element chain.

- The lattice $\mathbf{A}$ has a prime ideal.

The following corollary can be seen as a weak version of Theorem 2.5.

Corollary 3.3. The class of lattices without a prime ideal is closed under finite direct products.

The following example shows that even this weak version cannot be generalized to infinite products, not even if all factors are equal.

Example 3.4. (a) There are non-trivial lattices $\mathbf{M}$ such that no (finite or infinite) direct power of $\mathbf{M}$ has a prime ideal.

(b) On the other hand, there are lattices $\mathbf{A}$ without a prime ideal such that any infinite direct power $\mathbf{A}^{I}$ will contain a prime ideal.

Proof of (a). Let $\mathcal{M}$ be the class of all lattices of height 3 with at least 5 elements, i.e., the class of all bounded lattices $\mathbf{M}$ in which all elements except for $\sup \mathbf{M}$ and $\inf \mathbf{M}$ are incomparable. It is clear that no lattice in $\mathcal{M}$ has a prime ideal. The class $\mathcal{M}$ is closed under ultraproducts, since the property of being in $\mathcal{M}$ can be expressed by a first order statement. 
If $\mathbf{M}=\prod_{i \in I} \mathbf{M}_{i}$ is an arbitrary direct product with factors $\mathbf{M}_{i} \in \mathcal{M}$, and $h: \mathbf{M} \rightarrow \mathbf{C}$ is a homomorphism into a chain, then $h$ factors through an ultraproduct $\prod_{i \in I} \mathbf{M}_{i} \rightarrow \prod_{i \in I} \mathbf{M}_{I} / U \rightarrow \mathbf{C}, h=h^{\prime} \circ \kappa_{U}$. The map $h^{\prime}$ and therefore also $h$ must be constant.

Proof of (b). Let $\mathbf{A}$ be the lattice obtained from $\mathbb{N}=\{0,1,2, \ldots\}$ by replacing each odd number $2 k+1$ by a 3 -element antichain $a_{k}, b_{k}, c_{k}$, and each even number $2 k$ by a new element $d_{k}$. It is easy to see that $\mathbf{A}$ has no prime ideal.

We will show that every infinite power $\mathbf{A}^{I}$ contains a prime ideal. Clearly it is enough to show this for the case of countable $I$, say $I=\mathbb{N}$.

For any ultrafilter $U$ on $\mathbb{N}$ the following set $J_{U}$ is an ideal on $\prod_{i \in I} \mathbf{A}_{i}$ :

$$
J_{U}:=\left\{\left(x_{n}: n \in \mathbb{N}\right) \mid \exists k \exists C \in U \forall n \in C: x_{n} \leq d_{k}\right\}
$$

We now show that $J_{U}$ is a prime ideal. If

$$
\bar{x}=\left(x_{i}: i \in \mathbb{N}\right), \bar{y}=\left(y_{i}: i \in \mathbb{N}\right), \bar{z}=\left(z_{i}: i \in \mathbb{N}\right), \quad \bar{x} \wedge \bar{y}=\bar{z} \in J_{U},
$$

then there is some set $C \in U$ and some natural number $k \in \mathbb{N}$ such that $z_{n} \leq d_{k}$ holds for all $n \in C$. Now the two sets

$$
\left\{n: x_{n} \geq d_{k+1}\right\},\left\{n: y_{n} \geq d_{k+1}\right\}
$$

cannot both belong to $U$, as their intersection $D$ is disjoint to $C$. (Since $n \in D$ implies $x_{n} \wedge y_{n} \geq d_{k+1}$.)

Without loss of generality we have $\left\{n: x_{n} \leq d_{k+1}\right\} \in U$, so $\bar{x} \in J_{U}$.

\section{Acknowledgements}

Open access funding provided by TU Wien (TUW). We are grateful to the referee of a previous version of this paper for alerting us to [4], and to Gábor Czédli for suggesting the definition of HDI algebras.

Open Access. This article is distributed under the terms of the Creative Commons Attribution 4.0 International License (http://creativecommons.org/licenses/ by/4.0/), which permits unrestricted use, distribution, and reproduction in any medium, provided you give appropriate credit to the original author(s) and the source, provide a link to the Creative Commons license, and indicate if changes were made.

\section{References}

[1] Birkhoff, G.: Lattice Theory. Corr. Repr. of the 1967 3rd Edn. American Mathematical Society Colloquium Publications, vol. 25. American Mathematical Society (AMS), Providence (1979)

[2] Couceiro, M., Foldes, S., Meletiou, G.C.: On homomorphisms between products of median algebras. Algebra Universalis 78, 545-553 (2017)

[3] Couceiro, M., Marichal, J.-L., Teheux, B.: Conservative median algebras and semilattices. Order 33, 121-132 (2016) 
[4] Fraser, G.A., Horn, A.: Congruence relations in direct products. Proc. Am. Math. Soc. 26, 390-394 (1970)

[5] Grätzer, G.: Lattice Theory: Foundation. Birkhäuser, Basel (2011)

Ivan Chajda and Helmut Länger

Department of Algebra and Geometry

Palacký University Olomouc

17. listopadu 12

77146 Olomouc

Czech Republic

e-mail: ivan.chajda@upol.cz

Martin Goldstern and Helmut Länger

Institute of Discrete Mathematics and Geometry

TU Wien

Wiedner Hauptstraße 8-10

1040 Vienna

Austria

e-mail [M. Goldstern]: martin.goldstern@tuwien.ac.at

e-mail [H. Länger]: helmut.laenger@tuwien.ac.at

Received: 3 April 2017.

Accepted: 15 January 2018. 